\title{
OPEN Induced magnetic field and viscous dissipation on flows of two immiscible fluids in a rectangular channel
}

\begin{abstract}
Nehad Ali Shah ${ }^{1}$, Hussam Alrabaiah ${ }^{2,3}$, Dumitru Vieru $^{4}$ \& Se-Jin Yook ${ }^{5 \bowtie}$
The unsteady, magneto-hydrodynamic generalized Couette flows of two immiscible fluids in a rectangular channel with isothermal walls under the influence of an inclined magnetic field and an axial electric field have been investigated. Both fluids are considered electrically conducting and the solid boundaries are electrically insulated. Approximate analytical solutions for the velocity, induced magnetic, and temperature fields have been determined using the Laplace transform method along with the numerical Stehfest's algorithm for the inversion of the Laplace transforms. Also, for the nonlinear differential equation of energy, a numerical scheme based on the finite differences has been developed. A particular case has been numerically and graphically studied to show the evolution of the fluid velocity, induced magnetic field, and viscous dissipation in both flow regions.
\end{abstract}

The dynamics that occur in the motion of liquid-solid, liquid-liquid and liquid-gas environments are a rich source of interdisciplinary research, for example, in nanotechnology, the nuclear industry, as well as when cooling devices in microelectronics. The overall properties and applicability of multiphase systems are totally dependent on interface shapes, which influence and are influenced by each phase's flow area, where the heat fluxes are not equal since the transition in interfacial energy is considered ${ }^{1,2}$.

Magneto-hydrodynamic (MHD) devices, generators, accelerators, and flow meters use the flow and heat transfer of electrically conducting fluids in channels and circular pipes under the influence of a transverse magnetic field, and have uses in nuclear reactors, filtration, and heat exchangers, etc. Decades prior, there was an involvement in the influence of the outer magnetic field on heat-physical mechanisms. Blum et al. ${ }^{3}$ studied heat and mass transfer in the presence of a magnetic field and that was one of the first works. Many researchers have investigated the flow and heat transfer of a viscous, incompressible, electrically conducting fluid, between two infinite, parallel, insulating plates ${ }^{4-6}$. Convective heat transfer in channels has also become a common research subject in recent decades due to its applications in solar energy, gas cooled reactor protection, and crystal growth in liquids, among other things. The first research into a two-phase liquid metal magneto-fluid-mechanics generator was started by Thome ${ }^{7}$.In this study have been analyzed effects of the magnetic field on distribution of gas in the field direction, slip ratio, and two-phase pressure drop, by using streams of sodium-potassium alloy and nitrogen that were mixed and pumped through a vertical, rectangular channel with a transverse magnetic field applied perpendicularly to the long side of the cross section. Postlethwaite and Sluyter ${ }^{8}$ gave a summary of the heat transfer issues that MHD generators face. Lohrasbi and Sahai ${ }^{9}$ investigated MHD two-phase flow and heat transfer in a horizontal parallel-plate channel and identified analytical solutions for velocity and temperature profiles where only one of the fluids is electrically conducting. Closed-form solutions for two-phase flow and heat transfer in a horizontal channel with both phases electrically conducting were mentioned by Malashetty and Leela ${ }^{10,11}$.

Malashetty and Umavathi ${ }^{12}$ recently investigated two-phase MHD flow and heat transfer in an inclined channel with buoyancy effects for the case where only one of the phases is electrically conducting. The studies mentioned above are helpful in determining the impact of slag layers on the heat transfer aspects of coal fired MHD generators.

\footnotetext{
${ }^{1}$ Department of Mathematics, Lahore Leads University, Lahore, Pakistan. ${ }^{2}$ College of Engineering, Al Ain University, Al Ain, United Arab Emirates. ${ }^{3}$ Mathematics Department, Tafila Technical University, Tafila, Jordan. ${ }^{4}$ Department of Theoretical Mechanics, Technical University of lasi, lasi, Romania. ${ }^{5}$ School of Mechanical Engineering, Hanyang University, 222 Wangsimni-ro, Seongdong-gu, Seoul 04763, Republic of Korea. ${ }^{\square}$ email: ysjnuri@hanyang.ac.kr
} 


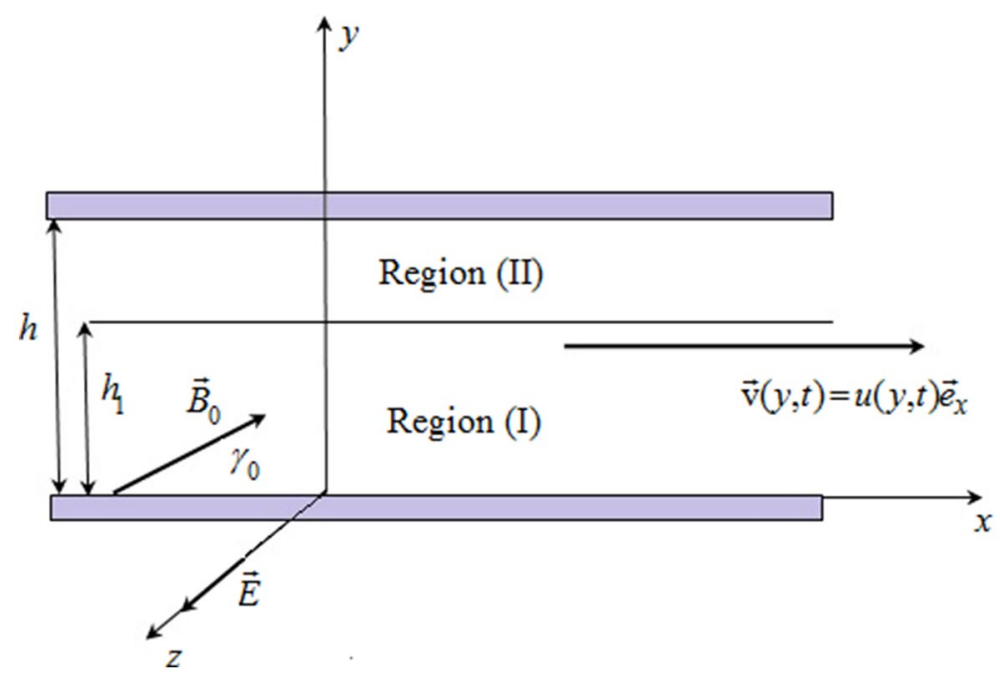

Figure 1. Physical model. Coordinate system.

Sai et al. ${ }^{13}$ investigated the flow of two immiscible fluids in a channel with one permeable wall caused by an oscillatory pressure gradient. They found slip conditions on the channel wall but assumed continuous velocities and shear stresses at the fluid-fluid interface. The authors studied the flow of water and mercury under the influence of a sinusoidal time-dependent pressure gradient as a particular case. By introducing cross terms to the generalized Darcy law, Pasquier et al. ${ }^{14}$ developed a statistical model of creeping flows that provides an explicit coupling term in both phases. Using an extension of the Buckley-Leverett theorem, the effect of the additional terminology on saturation profiles and pressure drops was studied. Nicodijevic et al. ${ }^{15}$ have studied magnetohydrodynamic Couette flows of two immiscible, electrically conducting Newtonian fluids in a horizontal channel. The flow is influenced by an electric field and an inclined magnetic field while, the channel walls are isothermal and insulated. The induced magnetic field has been also determined. Other interesting results of immiscible fluids flow can be found in ${ }^{16-18}$.

Thermal management systems/devices must be able to withstand a variety of thermal boundary conditions due to their vast range of applications in various sectors. Uniform heat flux, uniform wall temperature, and insulated wall boundary conditions are common examples. Numerous studies on studying the convective heat transfer process under such boundary circumstances have been published in the literature. However, depending on the flow environment, an asymmetric thermal boundary condition may be required to investigate the system's underlying thermal transport properties. We'd like to point out that there are a few studies in the literature that analyze the thermo-hydrodynamics of both Newtonian and non-Newtonian fluids while taking the aforementioned thermal boundary conditions into account ${ }^{19-21}$.

The purpose of this article it to investigate the unsteady, magneto-hydrodynamic generalized Couette flows of two immiscible fluids in a rectangular channel with isothermal walls under the influence of an inclined magnetic field and an axial electric field have been investigated. Both fluids are considered electrically conducting and the solid boundaries are electrically insulated. The problem is formulated into the dimensionless form and, at the interface the velocity, shear stress, induced magnetic and temperature fields are considered continuous functions. The bottom wall of channel is fixed, while the upper wall is moving with a given time-dependent velocity. The nonslip conditions on the solid boundaries are also considered. Approximate analytical solutions for the velocity, induced magnetic, and temperature fields have been determined using the Laplace transform method along with the numerical Stehfest's algorithm for the inversion of the Laplace transforms. Also, for the nonlinear differential equation of energy, a numerical scheme based on the finite differences has been developed. A particular case characterized by a time-exponential velocity of the upper wall, has been numerically and graphically studied to show the evolution of the fluid velocity, induced magnetic field, and viscous dissipation in both flow regions.

\section{Problem formulation}

The physical model shown in Fig. 1 consists of two infinite, parallel plates extending in the x-direction, and $\mathrm{z}$-direction. The $\mathrm{y}$-axis is perpendicular to the channel walls. The fluids in the two domains are assumed immiscible and electrically conducting. The flow is incompressible, unsteady, one-dimensional, and fully developed.

The region $R_{1}=\left\{(x, y, z) \in \mathbb{R}^{3}, 0 \leq y \leq h_{1}\right\}$ is occupied by a fluid of viscosity $\mu_{1}$, density $\rho_{1}$, electrical conductivity $\sigma_{1}$, thermal conductivity $k_{1}$, specific heat capacity $c_{p 1}$, and the magnetic permittivity $\mu_{e 1}$.

The region $R_{2}=\left\{(x, y, z) \in \mathbb{R}^{3}, h_{1} \leq y \leq h\right\}$ is field by a different fluid of viscosity $\mu_{2}$, density $\rho_{2}$, electrical conductivity $\sigma_{2}$, thermal conductivity $k_{2}$, specific heat capacity $c_{p 2}$, and the magnetic permittivity $\mu_{e 2}$.

It is supposed that fluids have laminar, one-dimensional flow with the velocity fields.

$$
\vec{V}_{i}(y, t)=u_{i}(y, t) \vec{e}_{x}, \quad i=1,2
$$

where $\vec{e}_{x}$ is the unit vector along the x-direction. 
A uniform magnetic field of the strength $\mathrm{B}_{0}$ is applied such that the angle between vectors $\vec{e}_{x}$ and $\overrightarrow{\mathrm{B}}_{0}$ is equal to $\gamma_{0} \in(0, \pi / 2]$, therefore, the applied magnetics vector field is

$$
\overrightarrow{\mathrm{B}}_{0}=\mathrm{B}_{0} \cos \gamma_{0} \vec{e}_{x}+\mathrm{B}_{0} \sin \gamma_{0} \vec{e}_{y} .
$$

Due to the fluid motion, a magnetic field is induced along of the lines of motion, namely

$$
\overrightarrow{\mathrm{B}}_{1}=b(y, t) \vec{e}_{x}
$$

is induced along of the lines of motion.

The total magnetic field is given by

$$
\overrightarrow{\mathrm{B}}=\overrightarrow{\mathrm{B}}_{0}+\overrightarrow{\mathrm{B}}_{1}=\left(b(y, t)+\mathrm{B}_{0} \cos \gamma_{0}\right) \vec{e}_{x}+\mathrm{B}_{0} \sin \gamma_{0} \vec{e}_{y}
$$

The walls of the rectangular channel are kept at the constant temperature $T_{w_{0}}$ and $T_{w_{1}}$, respectively and the plates are electrically insulated.

Also, a time-dependent electric field

$$
\vec{E}=E_{0} \sin (\omega t) \vec{e}_{z}
$$

is applied to the fluids.

In the above hypotheses, the continuity equation $\nabla \cdot \vec{V}_{i}=0$ is satisfied by the velocity fields in (1).

The electro-magneto-hydrodynamic fluids flow problem is mathematically described by ${ }^{22-24}$ :

The momentum equation

$$
\rho\left(\frac{\partial \vec{V}}{\partial t}+(\vec{V} \cdot \nabla) \vec{V}\right)=-\nabla p+\vec{J} \times \vec{B}
$$

The constitutive equation

$$
S=\mu\left(\nabla \vec{V}+(\nabla \vec{V})^{T}\right)
$$

The magnetic induction equation

$$
\frac{\partial \vec{B}}{\partial t}-\nabla \times(\vec{V} \times \vec{B})-\frac{1}{\sigma \mu_{e}} \Delta \vec{B}=0
$$

The energy equation

$$
\rho c_{p}\left(\frac{\partial T}{\partial t}+\vec{V} \nabla T\right)=k \Delta T+\mu \phi+\frac{1}{\sigma} \vec{J}^{2}
$$

where, the dissipation function $\phi$ is given by

$$
\phi=2\left[\left(\frac{\partial u}{\partial x}\right)^{2}+\left(\frac{\partial v}{\partial y}\right)^{2}+\left(\frac{\partial w}{\partial z}\right)^{2}\right]+\left(\frac{\partial v}{\partial x}+\frac{\partial u}{\partial y}\right)^{2}+\left(\frac{\partial w}{\partial y}+\frac{\partial v}{\partial z}\right)^{2}+\left(\frac{\partial u}{\partial z}+\frac{\partial w}{\partial x}\right)^{2}-\frac{2}{3}(\nabla \vec{V})^{2}=\left(\frac{\partial u}{\partial y}\right)^{2},
$$

and the current density vector due to the magnetic field and electric field is

$$
\vec{J}=\sigma(\vec{E}+\vec{V} \times \vec{B})=\sigma\left(E_{0} \sin (\omega t)+\mathrm{B}_{0} \sin \gamma_{0} u(y, t)\right) \vec{e}_{z}
$$

The magnetic body force becomes

$$
\begin{aligned}
\vec{F}_{m}= & \vec{J} \times \vec{B}=-\sigma \mathrm{B}_{0} \sin \gamma_{0}\left(E_{0} \sin (\omega t)+\mathrm{B}_{0} \sin \left(\gamma_{0}\right) u(y, t)\right) \vec{e}_{x} \\
& +\sigma\left(b(y, t)+\mathrm{B}_{0} \cos \gamma_{0}\right)\left(E_{0} \sin (\omega t)+\mathrm{B}_{0} \sin \left(\gamma_{0}\right) u(y, t)\right) \vec{e}_{y} .
\end{aligned}
$$

In the case of $\vec{V}=u(y, t) \vec{e}_{x}$, the above equations reduce to

$$
\begin{gathered}
\rho \frac{\partial u(y, t)}{\partial t}=-\frac{\partial p}{\partial x}+\frac{\partial S_{x y}(y, t)}{\partial y}-\sigma \mathrm{B}_{0} \sin \gamma_{0}\left(E_{0} \sin (\omega t)+\mathrm{B}_{0} \sin \left(\gamma_{0}\right) u(y, t)\right), \\
0=-\frac{\partial p}{\partial y}+\sigma\left(b(y, t)+\mathrm{B}_{0} \cos \gamma_{0}\right)\left(E_{0} \sin (\omega t)+\mathrm{B}_{0} \sin \left(\gamma_{0}\right) u(y, t)\right), \\
S_{x y}(y, t)=\mu \frac{\partial u(y, t)}{\partial t},
\end{gathered}
$$




$$
\begin{gathered}
\frac{\partial b(y, t)}{\partial t}-\mathrm{B}_{0} \sin \gamma_{0} \frac{\partial u(y, t)}{\partial y}-\frac{1}{\sigma \mu_{e}} \frac{\partial^{2} b(y, t)}{\partial y^{2}}=0, \\
\rho c_{p}\left(\frac{\partial T}{\partial t}+u \frac{\partial T}{\partial x}\right)=k \Delta T+\mu\left(\frac{\partial u}{\partial y}\right)^{2}+\sigma\left(E_{0} \sin (\omega t)+\mathrm{B}_{0} \sin \gamma_{0} u\right)^{2} .
\end{gathered}
$$

In this paper, we shall study the problem for zero pressure gradient in the $\mathrm{x}$-direction namely $\frac{\partial p}{\partial x}=0$. Also, the Eq. (14) that gives the pressure gradient in the y-direction will not be discussed, because it is clear that if $b(y, t)$ and $\mathrm{u}(y, t)$ are known the variation of the pressure $p$ is easily determined.

The flow, induced magnetic and temperature field of the fluid in two regions are determined by the equations

$$
\begin{gathered}
\rho_{i} \frac{\partial u_{i}(y, t)}{\partial t}=\frac{\partial S_{x y}^{i}(y, t)}{\partial y}-\sigma_{i} \mathrm{~B}_{0} \sin \gamma_{0}\left(E_{0} \sin (\omega t)+\mathrm{B}_{0} \sin \left(\gamma_{0}\right) u_{i}(y, t)\right), \\
S_{x y}^{i}(y, t)=\mu_{i} \frac{\partial u_{i}(y, t)}{\partial y}, \\
\frac{\partial b_{i}(y, t)}{\partial t}-\frac{1}{\sigma_{i} \mu_{e i}} \frac{\partial^{2} b_{i}(y, t)}{\partial y^{2}}=\mathrm{B}_{0} \sin \gamma_{0} \frac{\partial u_{i}(y, t)}{\partial y}, \\
\rho_{i} c_{p i}\left(\frac{\partial T_{i}}{\partial t}+u_{i} \frac{\partial T_{i}}{\partial x}\right)=k_{i} \Delta T_{i}+\mu_{i}\left(\frac{\partial u_{i}}{\partial y}\right)^{2}+\sigma_{i}\left(E_{0} \sin (\omega t)+\mathrm{B}_{0} \sin \gamma_{0} u_{i}\right)^{2} ; \quad i=1,2 .
\end{gathered}
$$

Along with Eqs. (18)-(21), the following initial, boundary and interface conditions are considered:

$$
\begin{gathered}
u_{1}(y, 0)=0, u_{2}(y, 0)=0, b_{1}(y, 0)=b_{2}(y, 0)=0, T_{1}(x, y, 0)=T_{w_{o}}=T_{2}(x, y, 0), \\
\begin{array}{c}
u_{1}(0, t)=0, u_{2}(h, t)=U_{0} f_{2}(t), U_{0}>0,(\text { No slip conditions }), \\
u_{1}\left(h_{1}, t\right)=u_{2}\left(h_{1}, t\right), \\
S_{x y}^{1}\left(h_{1}, t\right)=S_{x y}^{2}\left(h_{1}, t\right), \\
b_{1}(0, t)=0, \mathrm{~b}_{2}(h, t)=0, \\
b_{1}\left(h_{1}, t\right)=\mathrm{b}_{2}\left(h_{1}, t\right), \\
\frac{\left.\partial b_{1}(y, t)\right|_{y=h_{1}}}{\partial y}=\left.\frac{1}{\sigma_{2} \mu_{e 2}} \frac{\partial b_{2}(y, t)}{\partial y}\right|_{y=h_{1}} \\
T_{1}(x, 0, t)=T_{w_{0}}, \mathrm{~T}_{2}(x, h, t)=T_{w_{1}} \\
T_{1}\left(x, h_{1}, t\right)=\mathrm{T}_{2}\left(x, h_{1}, t\right), \\
\left.\frac{\partial T_{1}}{\partial y}\right|_{y=h_{1}}=\left.k_{2} \frac{\partial T_{2}}{\partial y}\right|_{y=h_{1}}
\end{array}
\end{gathered}
$$

In the present problem, it has assumed that the two plates are maintained at constant temperatures. Under the flow's assumptions, the temperature fields can be considered independent of the longitudinal variable $\mathrm{x}$. The term involving $\frac{\partial T}{\partial x}=0$ in the energy Eq. (21) drops out for such conditions.

We introduce the non-dimensional entities

$$
\begin{aligned}
& \tilde{u}_{i}=\frac{u_{i}}{U_{0}}, \tilde{\mathrm{y}}=\frac{y}{h_{1}}, \tilde{\mathrm{x}}=\frac{x}{h_{1}}, \mathrm{~d}=\frac{h}{h_{1}}, \tilde{\mathrm{t}}=\frac{U_{0} t}{h_{1}}, \tilde{S}_{\tilde{x} \tilde{y}}^{i}=\frac{S_{x y}^{i}}{\rho_{i} U_{0}^{2}}, \tilde{\omega}=\frac{h_{1} \omega}{U_{0}}, E_{i}=\frac{\sigma_{i} B_{0} E_{0} h_{1} \sin \gamma_{0}}{\rho_{i} U_{0}^{2}}, \\
& F_{i}=\frac{\sigma_{i} B_{0}^{2} h_{1} \sin ^{2} \gamma_{0}}{\rho_{i} U_{i}}, \operatorname{Re}_{i}=\frac{h_{1} U_{0}}{v_{i}}, \tilde{b}_{i}=\frac{b_{i}}{B_{0} \sin \gamma_{0}}, \theta_{i}=\frac{T_{i}-T_{w_{0}}}{T_{w_{1}}-T_{w_{0}}}, \operatorname{Pr}_{i}=\frac{\mu_{i} c_{p i}}{k_{i}}, \mathrm{Ec}_{i}=\frac{U_{0}^{2}}{c_{p i}\left(T_{w_{1}}-T_{w_{0}}\right)}, \\
& H a_{i}=B_{0} h_{1} \sqrt{\frac{\sigma_{i}}{\mu_{i}}}, \mathrm{G}_{0}=\frac{E_{0}}{B_{0} U_{0} \sin \gamma_{0}}, \tilde{f}(\tilde{t})=f_{2}\left(\frac{h_{1}}{U_{0}} \tilde{t}\right), \delta_{0}=\frac{\mu_{e 1} \sigma_{1}}{\mu_{e 2} \sigma_{2}}, k_{0}=\frac{k_{2}}{k_{1}}, R m_{i}=U_{0} h_{1} \sigma_{i} \mu_{e i} .
\end{aligned}
$$


Equations (18)-(21) become (the notation “ " is neglected)

$$
\begin{aligned}
& \frac{\partial u_{i}(y, t)}{\partial t}=\frac{\partial S_{x y}^{i}(y, t)}{\partial y}-E_{i} \sin (\omega t)-F_{i} u_{i}(y, t), \\
& S_{x y}^{i}(y, t)=\frac{1}{\operatorname{Re}_{i}} \frac{\partial u_{i}(y, t)}{\partial y}, \\
& \frac{\partial b_{i}(y, t)}{\partial t}-\frac{1}{R m_{i}} \frac{\partial^{2} b_{i}(y, t)}{\partial y^{2}}=\frac{\partial u_{i}(y, t)}{\partial y}
\end{aligned}
$$

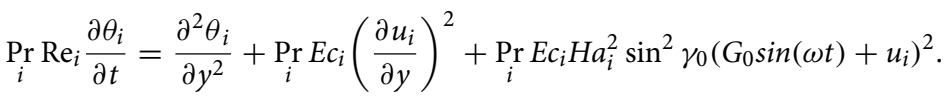

The non-dimensional boundary conditions are

$$
\begin{gathered}
u_{i}(y, 0)=0, b_{i}(y, 0)=0, \theta_{i}(y, 0)=0, i=1,2, y \in[0, d] \\
u_{1}(0, t)=0, u_{2}(d, t)=f(t) \\
u_{1}(1, t)=u_{2}(1, t) \\
S_{x y}^{1}(1, t)=S_{x y}^{2}(1, t) \\
b_{1}(0, t)=0, b_{2}(d, t)=0 \\
b_{1}(1, t)=b_{2}(1, t), \\
\left.\frac{\partial b_{1}(y, t)}{\partial y}\right|_{y=1}=\left.\delta_{0} \frac{\partial b_{2}(y, t)}{\partial y}\right|_{y=1} \\
\theta_{1}(0, t)=0, \theta_{2}(d, t)=1, \\
\theta_{1}(1, t)=\theta_{2}(1, t), \\
\left.\frac{\partial \theta_{1}(y, t)}{\partial y}\right|_{y=1}=\left.k_{0} \frac{\partial \theta_{2}(y, t)}{\partial y}\right|_{y=1} .
\end{gathered}
$$

\section{Velocity field distribution}

To solve Eqs. (33) and (34) along with initial-boundary conditions (37) and (38), we use the Laplace transform method. Applying the Laplace transform to Eqs. (33) and (34), we obtain

$$
\begin{gathered}
s \bar{u}_{i}(y, s)=\frac{\partial \bar{S}_{x y}^{i}(y, t)}{\partial y}-E_{i} \frac{\omega}{s^{2}+\omega^{2}}-F_{i} \bar{u}_{i}(y, s), \\
\bar{S}_{x y}^{i}(y, s)=\frac{1}{\operatorname{Re}_{i}} \frac{\partial \bar{u}_{i}(y, s)}{\partial y},
\end{gathered}
$$

where, $\bar{\chi}(y, s)=\int_{0}^{\infty} \chi(y, t) \exp (-s t) d s$ denotes the Laplace transform of function $\chi(y, t)$.

Eliminating $\bar{S}_{x y}^{i}$ between Eqs. (41) and (42), we obtain the following equation for the Laplace transform $\bar{u}_{i}(y, s)$ of the velocity $u_{i}(y, t), i=1,2$ :

$$
\frac{\partial^{2} \bar{u}_{i}(y, s)}{\partial y^{2}}=\operatorname{Re}_{i}\left(s+F_{i}\right) \bar{u}_{i}(y, s)+\frac{E_{i} \operatorname{Re}_{i} \omega}{s^{2}+\omega^{2}}, i=1,2 .
$$

Functions $\bar{u}_{i}(y, s)$ have to satisfy the boundary and interface conditions

$$
\begin{aligned}
& \bar{u}_{1}(0, s)=0, \bar{u}_{2}(d, s)=\bar{f}(s), \\
& \bar{u}_{1}(1, s)=\bar{u}_{2}(1, s), \\
& \left.\frac{1}{\operatorname{Re}_{1}} \frac{\partial \bar{u}_{1}(y, s)}{\partial y}\right|_{y=1}=\left.\frac{1}{\operatorname{Re}_{2}} \frac{\partial \bar{u}_{2}(y, s)}{\partial y}\right|_{y=1} .
\end{aligned}
$$

The general solution of Eq. (43) is

$$
\bar{u}_{i}(y, s)=C_{i 1}(s) \sinh \left(\bar{a}_{i}(s) y\right)+\bar{C}_{i 2}(s) \cosh \left(\bar{a}_{i}(s) y\right)+b_{i}(s),
$$


where

$$
\bar{a}_{i}(s)=\sqrt{\operatorname{Re}_{i}\left(s+F_{i}\right)}, b_{0 i}(s)=\frac{E_{i} \omega}{\left(s+F_{i}\right)\left(s^{2}+\omega^{2}\right)}, i=1,2 .
$$

Using (44), the following expressions of the functions $C_{i 1}(s), C_{i 2}(s), i=1,2$ are found:

$$
\begin{aligned}
& \bar{C}_{11}(s)=\frac{\left(\bar{f}(s)-\bar{b}_{02}(s)\right) \cosh \left(\bar{a}_{2}(s)\right)}{\cosh \left(\bar{a}_{2}(s) d\right) \sinh \left(\bar{a}_{1}(s)\right)}+\frac{\bar{b}_{02}(s)-\bar{b}_{01}(s)+\bar{b}_{01}(s) \cosh \left(\bar{a}_{1}(s)\right)}{\sinh \left(\bar{a}_{1}(s)\right)} \\
& -\frac{\sinh \left((d-1) \bar{a}_{2}(s)\right)}{\cosh \left(\bar{a}_{2}(s) d\right) \sinh \left(\bar{a}_{1}(s)\right)} \bar{C}_{21}(s), \\
& \bar{C}_{12}(s)=-b_{01}(s) \text {, } \\
& \bar{C}_{22}(s)=\frac{\bar{f}(s)-\bar{b}_{02}(s)}{\cosh \left(\bar{a}_{2}(s) d\right)}-\frac{\sinh \left(\bar{a}_{2}(s) d\right)}{\cosh \left(\bar{a}_{2}(s) d\right)} \bar{C}_{21}(s), \\
& \bar{C}_{21}=\frac{F a(s)}{F b(s)}, \\
& F a(s)=\operatorname{Re}_{2} \bar{a}_{1}(s)\left[\bar{b}_{01}(s) \cosh \left(\bar{a}_{2}(s) d\right)+\left(\bar{f}(s)-\bar{b}_{02}(s)\right) \cosh \left(\bar{a}_{1}(s)\right) \cosh \left(\bar{a}_{2}(s)\right)\right. \\
& \left.+\left(\bar{b}_{02}(s)-\bar{b}_{01}(s)\right) \cosh \left(\bar{a}_{1}(s)\right) \cosh \left(\bar{a}_{2}(s) d\right)\right]-\operatorname{Re}_{1} \bar{a}_{2}(s)\left(\bar{f}(s)-\bar{b}_{02}(s)\right) \sinh \left(\bar{a}_{1}(s)\right) \sinh \left(\bar{a}_{2}(s)\right), \\
& F b(s)=\operatorname{Re}_{1} \bar{a}_{2}(s) \cosh \left(\bar{a}_{1}(s)\right) \sinh \left[(d-1) \bar{a}_{2}(s)\right] \\
& +\operatorname{Re}_{2} \bar{a}_{1}(s) \sinh \left(\bar{a}_{1}\right) \cosh \left[(d-1) \bar{a}_{2}(s)\right] \text {. }
\end{aligned}
$$

Although the inverse Laplace transform of the function (45) can be obtained using the residue theorem, the analytical expression of the $u(y, t)$ is complicated and difficult to be used for numerical evaluation. For this reason, we prefer to obtain the numerical values of the inverse Laplace transform of $\bar{u}(y, s)$ using Stehfest's numerical algorithm ${ }^{25,26}$

According to the Stehfest's algorithm, the approximate values of function $u_{i}(y, t)$ are given by

$$
u_{i}(y, t) \simeq \frac{\ln 2}{t} \sum_{k=1}^{M} b_{k} \bar{u}_{i}\left(y, \frac{k \ln 2}{t}\right)
$$

where

$$
b_{k}=(-1)^{k+\frac{M}{2}} \sum_{j=\left[\frac{k+1}{2}\right]}^{\min \left(k, \frac{M}{2}\right)} \frac{j^{M / 2}(2 j)}{(M / 2-j) ! j !(j-1) !(k-j) !(2 j-k) !} .
$$
$x$.

In the above relation $M$ is an even, positive integer number and $[x]$ denotes the integer part of the real number

\section{The induced magnetic field}

The magnetic field induction is given by Eq. (35) along with conditions (39). Applying the Laplace transform to Eq. (35), using initial conditions $(37)_{2}$ and the velocity expression (45), we obtain for the Laplace transform $\bar{b}_{i}(y, s)$ of $b_{i}(y, t)$ the differential equation

$$
\frac{\partial^{2} \bar{b}_{i}(y, s)}{\partial y^{2}}-R m_{i} s \bar{b}_{i}(y, s)=-R m_{i} \bar{a}_{i}(s)\left[\bar{C}_{i 1}(s) \cosh \left(\bar{a}_{i}(s) y\right)+\bar{C}_{i 2}(s) \sinh \left(\bar{a}_{i}(s) y\right)\right],
$$

along with the boundary and interface conditions

$$
\begin{aligned}
& \bar{b}_{1}(0, s)=0, \bar{b}_{2}(d, s)=0, \\
& \bar{b}_{1}(1, s)=\bar{b}_{2}(1, s), \\
& \left.\frac{\partial \bar{b}_{1}(y, s)}{\partial y}\right|_{y=1}=\left.\delta_{0} \frac{\partial \bar{b}_{2}(y, s)}{\partial y}\right|_{y=1} .
\end{aligned}
$$

The general solution of Eq. (50) is given by

$$
\begin{aligned}
\bar{b}_{i}(y, s)= & \bar{D}_{i 1}(s) \sinh \left(y \sqrt{R m_{i} s}\right)+\bar{D}_{i 2}(s) \cosh \left(y \sqrt{R m_{i} s}\right) \\
& +\bar{d}_{i}(s)\left[\bar{C}_{i 1}(s) \cosh \left(\bar{a}_{i}(s) y\right)+\bar{C}_{i 2}(s) \sinh \left(\bar{a}_{i}(s) y\right)\right],
\end{aligned}
$$

where 


$$
\bar{d}_{i}(s)=\frac{R m_{i} \bar{a}_{i}(s)}{R m_{i} s-\bar{a}_{i}^{2}(s)}=\frac{R m_{i} \sqrt{\operatorname{Re}_{i}\left(s+F_{i}\right)}}{\left(R m_{i}-\operatorname{Re}_{i}\right) s-\operatorname{Re}_{i} F_{i}} .
$$

We introduce the notations

$$
\begin{gathered}
\bar{G}_{i}(y, s)=\bar{d}_{i}(s)\left[\bar{C}_{i 1}(s) \cosh \left(\bar{a}_{i}(s) y\right)+\bar{C}_{i 2}(s) \sinh \left(\bar{a}_{i}(s) y\right)\right] \\
\bar{H}_{i}(y, s)=\frac{\partial \bar{G}_{i}(y, s)}{\partial y}=\bar{a}_{i}(s) \bar{d}_{i}(s)\left[\bar{C}_{i 1}(s) \sinh \left(\bar{a}_{i}(s) y\right)+\bar{C}_{i 2}(s) \cosh \left(\bar{a}_{i}(s) y\right)\right],
\end{gathered}
$$

we have

$$
\begin{aligned}
\bar{b}_{1}(y, s) & =\bar{D}_{i 1}(s) \sinh \left(y \sqrt{R m_{i} s}\right)+\bar{D}_{i 2}(s) \sinh \left(y \sqrt{R m_{i} s}\right)+\bar{G}_{i}(y, s), \\
\frac{\partial \bar{b}_{1}(y, s)}{\partial y} & =\sqrt{R m_{i} s}\left[\bar{D}_{i 1}(s) \cosh \left(y \sqrt{R m_{i} s}\right)+\bar{D}_{i 2}(s) \sinh \left(y \sqrt{R m_{i} s}\right)\right]+\bar{H}_{i}(y, s) .
\end{aligned}
$$

Using the boundary and interface conditions (51), we obtain the algebraic system for the unknown functions $\bar{D}_{i 1}(s), \bar{D}_{i 1}(s), i=1,2$ :

$$
\begin{aligned}
& \bar{D}_{12}(s)+\bar{G}_{1}(0, s)=0, \\
& \bar{D}_{21}(s) \sinh \left(d \sqrt{R m_{2} s}\right)+\bar{D}_{22}(s) \cosh \left(d \sqrt{R m_{2} s}\right)+\bar{G}_{2}(d, s)=0, \\
& \bar{D}_{11}(s) \sinh \left(\sqrt{R m_{1} s}\right)+\bar{D}_{12}(s) \cosh \left(\sqrt{R m_{1} s}\right)+\bar{G}_{1}(1, s) \\
& \quad=\bar{D}_{21}(s) \sinh \left(\sqrt{R m_{2} s}\right)+\bar{D}_{22}(s) \cosh \left(\sqrt{R m_{2} s}\right)+\bar{G}_{2}(1, s), \\
& \sqrt{R m_{1} s}\left[\bar{D}_{11}(s) \cosh \left(\sqrt{R m_{1} s}\right)+\bar{D}_{12}(s) \sinh \left(\sqrt{R m_{1} s}\right)\right]+\bar{H}_{1}(1, s) \\
& \quad=\delta_{0} \sqrt{R m_{2} s}\left[\bar{D}_{21}(s) \cosh \left(\sqrt{R m_{2} s}\right)+\bar{D}_{22}(s) \sinh \left(\sqrt{R m_{2} s}\right)\right]+\delta_{0} \bar{H}_{2}(1, s) .
\end{aligned}
$$

whose solution is

$$
\begin{gathered}
\bar{D}_{12}(s)=-\bar{G}_{1}(0, s)=-\bar{d}_{1}(s) \bar{C}_{11}(s), \\
\bar{D}_{22}(s)=\frac{-\bar{G}_{2}(d, s)}{\cosh \left(d \sqrt{R m_{2} s}\right)}-\frac{\sinh \left(d \sqrt{R m_{2} s}\right)}{\cosh \left(d \sqrt{R m_{2} s}\right)} \bar{D}_{21}(s), \\
\bar{D}_{11}(s)=\bar{D}_{00}(s)+\bar{D}_{01}(s) \bar{D}_{21}(s)
\end{gathered}
$$

where

$$
\begin{aligned}
& \bar{D}_{00}(s)=\bar{G}_{1}(1, s) \frac{\cosh \left(\sqrt{R m_{1} s}\right)}{\sinh \left(\sqrt{R m_{1} s}\right)}+\frac{\bar{G}_{2}(1, s)-\bar{G}_{1}(1, s)}{\sinh \left(\sqrt{R m_{1} s}\right)}-\frac{\bar{G}_{2}(d, s) \cosh \left(\sqrt{R m_{2} s}\right)}{\sinh \left(\sqrt{R m_{1} s}\right) \cosh \left(d \sqrt{R m_{2} s}\right)}, \\
& \bar{D}_{01}(s)=\frac{\sinh \left(\sqrt{R m_{2} s}\right) \cosh \left(d \sqrt{R m_{2} s}\right)-\sinh \left(d \sqrt{R m_{2} s}\right)}{\sinh \left(\sqrt{R m_{1} s}\right) \cosh \left(d \sqrt{R m_{2} s}\right)}
\end{aligned}
$$

$$
\bar{D}_{21}(s)=\frac{\bar{E}_{01}(s)}{\bar{E}_{00}(s)}
$$

with

$$
\begin{aligned}
\bar{E}_{00}(s)= & \bar{D}_{01} \sqrt{R m_{1} s} \cosh \left(\sqrt{R m_{1} s}\right)-\delta_{0} \sqrt{R m_{2} s} \frac{\cosh \left[(d-1) \sqrt{R m_{2} s}\right]}{\cosh \left(d \sqrt{R m_{2} s}\right)}, \\
\bar{E}_{01}(s)= & \sqrt{R m_{1} s} \sinh \left(\sqrt{R m_{1} s}\right) \bar{G}_{1}(0, s)-\bar{H}_{1}(1, s)+\delta_{0} \bar{H}_{2}(1, s) \\
& -\sqrt{R m_{1} s} \cosh \left(\sqrt{R m_{1} s}\right) \bar{D}_{00}(s)-\frac{\delta_{0} \sqrt{R_{m 2} s} \sinh \left(\sqrt{R_{m 2} s}\right) \bar{G}_{2}(d, s)}{\cosh \left(d \sqrt{R_{m 2} s}\right)} .
\end{aligned}
$$

Numerical values of the induced magnetic fields (52) shall be determined with the numerical approximate formula (48) updated for $b_{i}(y, t)$.

The temperature field

Approximate analytical solution. Let's introduce notation

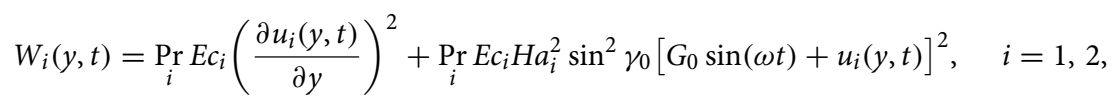


where $u_{i}$ is given by Eq. (45).

Temperature fields are solutions of the equations

$$
\frac{\partial^{2} \theta_{i}(y, t)}{\partial y^{2}}-\operatorname{Pr}_{i} \operatorname{Re}_{i} \frac{\partial \theta_{i}(y, t)}{\partial t}+W_{i}(y, t)=0, \quad i=1,2,
$$

along with the initial-boundary and interface conditions

$$
\begin{gathered}
\theta_{i}(y, 0)=0, y \in[0, d], \\
\theta_{1}(0, t)=0, \quad \theta_{2}(d, t)=1, \quad t>0, \\
\theta_{1}(1, t)=\theta_{2}(1, t)=1, \quad t>0, \\
\left.\frac{\partial \theta_{1}(y, t)}{\partial y}\right|_{y=1}=\left.k_{0} \frac{\partial \theta_{2}(y, t)}{\partial y}\right|_{y=1}, \quad t>0 .
\end{gathered}
$$

Applying the Laplace transform to Eq. (65), we obtain the transformed equation

$$
\frac{\partial^{2} \bar{\theta}_{i}(y, s)}{\partial y^{2}}-Q_{i} \bar{s}_{i}(y, s)+\bar{W}_{i}(y, s)=0, \quad i=1,2, \quad Q_{i}=\underset{i}{\operatorname{Pr} \operatorname{Re}_{i}}
$$

The general solution of Eq. (70) is

$$
\bar{\theta}_{i}(y, s)=\bar{D}_{i 1}(y, s) e^{-y \sqrt{Q_{i} s}}+\bar{D}_{i 2}(y, s) e^{y \sqrt{Q_{i} s}}
$$

where $\bar{D}_{i 1}, \bar{D}_{i 2}$ have to satisfy conditions

$$
\begin{aligned}
& e^{-y \sqrt{Q_{i} s}} \bar{D}_{i 1}^{\prime}(y, s)+e^{y \sqrt{Q_{i} s}} \bar{D}_{i 2}^{\prime}(y, s)=0, \\
& -\sqrt{Q_{i} s} e^{-y \sqrt{Q_{i}} s} \bar{D}_{i 1}^{\prime}(y, s)+\sqrt{Q_{i} s} e^{y \sqrt{Q_{i} s}} \bar{D}_{i 2}^{\prime}(y, s)=-\bar{W}_{i}(y, s) .
\end{aligned}
$$

From Eq. (72), we have

$$
\bar{D}_{i 1}^{\prime}(y, s)=\frac{e^{y \sqrt{Q_{i} s}}}{2 \sqrt{Q_{i} s}} \bar{W}_{i}(y, s) ; \bar{D}_{i 2}^{\prime}(y, s)=\frac{-e^{-y \sqrt{Q_{i} s}}}{2 \sqrt{Q_{i} s}} \bar{W}_{i}(y, s),
$$

respectively,

$$
\bar{D}_{i 1}(y, s)=\int_{0}^{y} \frac{e^{\xi \sqrt{Q_{i} s}}}{2 \sqrt{Q_{i} s}} \bar{W}_{i}(\xi, s) d \xi+\bar{E}_{i 1}(s), \bar{D}_{i 2}(y, s)=-\int_{0}^{y} \frac{e^{-\xi \sqrt{Q_{i} s}}}{2 \sqrt{Q_{i} s}} \bar{W}_{i}(\xi, s) d \xi+\bar{E}_{i 2}(s) .
$$

Replacing (74) into (71), we obtain

$$
\bar{\theta}_{i}(y, s)=\bar{E}_{i 1}(s) e^{-y \sqrt{Q_{i} s}}+\bar{E}_{i 2}(s) e^{y \sqrt{Q_{i} s}}+e^{-y \sqrt{Q_{i} s}} \bar{\Phi}_{i 1}(y, s)+e^{y \sqrt{Q_{i} s}} \bar{\Phi}_{i 2}(y, s),
$$

where,

$$
\bar{\Phi}_{i 1}(y, s)=\int_{0}^{y} \frac{e^{\xi \sqrt{Q_{i} s}}}{2 \sqrt{Q_{i} s}} \bar{W}_{i}(\xi, s) d \xi, \bar{\Phi}_{i 2}(y, s)=-\int_{0}^{y} \frac{e^{-\xi \sqrt{Q_{i} s}}}{2 \sqrt{Q_{i} s}} \bar{W}_{i}(\xi, s) d \xi .
$$

Unknown functions $\bar{E}_{i 1}(s), \bar{E}_{i 2}(s)$ shall be determined using the boundary and interface conditions

$$
\begin{aligned}
& \bar{\theta}_{1}(0, s)=0, \bar{\theta}_{2}(d, s)=\frac{1}{s} \\
& \bar{\theta}_{1}(1, s)=\bar{\theta}_{2}(1, s) \\
& \left.\frac{\partial \bar{\theta}_{1}(y, s)}{\partial y}\right|_{y=1}=\left.k_{0} \frac{\partial \bar{\theta}_{2}(y, s)}{\partial y}\right|_{y=1} .
\end{aligned}
$$

After direct calculations, we obtain

$$
\bar{E}_{12}(s)=-\bar{E}_{11}(s), \quad \bar{E}_{21}(s)=\frac{1}{s} e^{d \sqrt{Q_{2} s}}-\bar{\Phi}_{21}(d, s)-e^{2 d \sqrt{Q_{2} s}} \bar{\Phi}_{22}(d, s)-e^{2 d \sqrt{Q_{2} s}} \bar{E}_{22}(s),
$$




$$
\begin{gathered}
\bar{E}_{11}=\frac{-1}{2} \frac{\bar{G}_{1}(s) \sqrt{Q_{2} s} \cosh \left[(d-1) \sqrt{Q_{2} s}\right]+\bar{G}_{2}(s) \sinh \left[(d-1) \sqrt{Q_{2} s}\right]}{\sqrt{Q_{2} s} \sinh \left(\sqrt{Q_{1} s}\right) \cosh \left[(d-1) \sqrt{Q_{2} s}\right]+\sqrt{Q_{1} s} \cosh \left(\sqrt{Q_{1} s}\right) \sinh \left[(d-1) \sqrt{Q_{2} s}\right]}, \\
\bar{E}_{22}=\frac{1}{2} \frac{\bar{G}_{1}(s) \sqrt{Q_{1} s} \cosh \left(\sqrt{Q_{1} s}\right)-\bar{G}_{2}(s) \sinh \left(\sqrt{Q_{1} s}\right)}{e^{d \sqrt{Q_{2} s}}\left\{\sqrt{Q_{2} s} \sinh \left(\sqrt{Q_{1} s}\right) \cosh \left[(d-1) \sqrt{Q_{2} s}\right]+\sqrt{Q_{1} s} \cosh \left(\sqrt{Q_{1} s}\right) \sinh \left[(d-1) \sqrt{Q_{2} s}\right]\right\}},
\end{gathered}
$$

where,

$$
\begin{aligned}
\bar{G}_{1}(s)= & \frac{1}{s} e^{(d-1) \sqrt{Q_{2} s}}-e^{-\sqrt{Q_{2} s}} \bar{\Phi}_{21}(d, s)-e^{(2 d-1) \sqrt{Q_{2} s}} \bar{\Phi}_{22}(d, s)+e^{-\sqrt{Q_{2} s}} \bar{\Phi}_{21}(1, s)+e^{\sqrt{Q_{2} s}} \bar{\Phi}_{22}(1, s), \\
\bar{G}_{2}(s)= & -\frac{\sqrt{Q_{2} s}}{s} e^{(d-1) \sqrt{Q_{2} s}}+\sqrt{Q_{2} s} e^{-\sqrt{Q_{2} s}} \bar{\Phi}_{21}(d, s)+\sqrt{Q_{2} s} e^{(2 d-1) \sqrt{Q_{2} s}} \bar{\Phi}_{22}(d, s) \\
& -\sqrt{Q_{2} s} e^{-\sqrt{Q_{2} s}} \bar{\Phi}_{21}(1, s)+\sqrt{Q_{2} s} e^{\sqrt{Q_{2} s}} \bar{\Phi}_{22}(1, s)+\sqrt{Q_{1} s} e^{-\sqrt{Q_{1} s}} \bar{\Phi}_{11}(1, s)-\sqrt{Q_{1} s} e^{\sqrt{Q_{1} s}} \bar{\Phi}_{12}(1, s) .
\end{aligned}
$$

Numerical values of the temperature $\theta_{i}(y, t)$ could be determined with the numerical approximate formula (48) updated for $\bar{\theta}_{i}(y, s)$. However, the expressions of the temperature fields (75) are quite complicated and also contain functions $\Phi_{i 1}(s), \Phi_{i 2}(s)$ that are defined by means of some definite integrals. For this reason, the numerical inversion with the Stehfest's algorithm becomes difficult. Therefore, in the following, we will elaborate a numerical scheme adequate to the integration of the differential Eq. (65) with the initial-boundary conditions (66)-(69).

Numerical scheme. Temperature fields $\theta_{1}(y, t), \theta_{2}(y, t)$ are solutions of the equations

$$
\begin{aligned}
& Q_{1} \frac{\partial \theta_{1}(y, t)}{\partial t}-\frac{\partial^{2} \theta_{1}(y, t)}{\partial t^{2}}-W_{1}(y, t)=0, \\
& Q_{2} \frac{\partial \theta_{2}(y, t)}{\partial t}-\frac{\partial^{2} \theta_{2}(y, t)}{\partial t^{2}}-W_{2}(y, t)=0,
\end{aligned}
$$

along with the initial, boundary and interface conditions

$$
\begin{gathered}
\theta_{1}(y, 0)=0, y \in[0,1] ; \theta_{2}(y, 0)=0, y \in[1, d] \\
\theta_{1}(0, t)=0 ; \theta_{2}(d, t)=1, t>0 \\
\theta_{1}(1, t)=\theta_{2}(1, t)=0, t>0 \\
\left.\frac{\partial \theta_{1}(y, t)}{\partial y}\right|_{y=1}=\left.\frac{\partial \theta_{2}(y, t)}{\partial y}\right|_{y=1} .
\end{gathered}
$$

We consider the step size $\Delta t$ for the time $\mathrm{t}$, step size $\Delta y_{1}$ for the spatial coordinate $y \in[0,1]$, and the sequences $t_{n}=n \Delta t, n=0,1, \ldots, N_{t}, y_{j}=j \Delta y_{1}, j=0,1, \ldots, N_{1}, \Delta t=T / N_{t}, \Delta y_{1}=1 / N_{1}$. Using the approximate formulas for the derivatives,

$$
\begin{aligned}
& \left.\frac{\partial \chi(y, t)}{\partial t}\right|_{(y, t)=\left(y_{j}, t_{n}\right)}=\frac{1}{\Delta t}\left[\chi\left(y_{j}, t_{n+1}\right)-\chi\left(y_{j}, t_{n}\right)\right], n=0,1, \ldots, N_{t}-1, j=0,1, \ldots, N_{1}, \\
& \left.\frac{\partial^{2} \chi(y, t)}{\partial y^{2}}\right|_{(y, t)=\left(y_{j}, t_{n}\right)}=\frac{1}{\Delta y^{2}}\left[\chi\left(y_{j+1}, t_{n}\right)-2 \chi\left(y_{j}, t_{n}\right)+\chi\left(y_{j-1}, t_{n}\right)\right], n=0,1, \ldots, N_{t}, j=1,2, \ldots N_{1}-1,
\end{aligned}
$$

Equation $(82)_{1}$ is written as

$$
\theta_{1}^{j, n+1}=\alpha_{00} \theta_{1}^{j+1, n}+\alpha_{01} \theta_{1}^{j, n}+\alpha_{02} \theta_{1}^{j-1, n}+\alpha_{03} W_{1}^{j, n}, n=0,1, \ldots, N_{t}-1, j=1,2, \ldots, N_{1}-1,
$$

where,

$$
\theta_{1}^{j, n}=\theta_{1}\left(y_{j}, t_{n}\right), \text { and, } \alpha_{00}=\alpha_{02}=\frac{\Delta t}{Q_{1} \Delta y_{1}^{2}}, \alpha_{01}=1-\frac{2 \Delta t}{Q_{1} \Delta y_{1}^{2}}, \alpha_{03}=\frac{\Delta t}{Q_{1}} .
$$

The initial and boundary conditions (83) and (84) lead to the following relations:

$$
\theta_{1}^{j, 0}=0, j=0,1, \ldots, N_{1} ; \quad \theta_{1}^{0, n}=0, n=0,1, \ldots, N_{t} .
$$

For the interval $y \in[1, d]$, we consider the sequence

$$
y_{N_{1}}=1, y_{N_{1}+k}=1+k \Delta y_{2}, \quad k=1,2, \ldots, N_{2}, \Delta y_{2}=\frac{d-1}{N_{2}} .
$$


From the interface condition $(85)_{1}$, we obtain

$$
\theta_{1}^{N_{1}, n}=\theta_{1}^{N_{1}, n}, \quad n=0,1, \ldots, N_{t},
$$

while, from the condition $(85)_{2}$, we have

$$
\begin{aligned}
& \theta_{1}^{N_{1}, n}=\theta_{2}^{N_{1}, n}=\delta_{00} \theta_{2}^{N_{1}+1, n}+\delta_{01} \theta_{1}^{N_{1}-1, n}, \quad n=1,2, \ldots, N_{t}, \\
& \delta_{00}=\frac{k_{0} \Delta y_{1}}{\Delta y_{2}+k_{0} \Delta y_{1}}, \delta_{01}=\frac{\Delta y_{2}}{\Delta y_{2}+k_{0} \Delta y_{1}} .
\end{aligned}
$$

Equation $(82)_{2}$ is written as

$$
\theta_{2}^{N_{1}+k, n+1}=\beta_{00} \theta_{2}^{N_{1}+k+1, n}+\beta_{01} \theta_{2}^{N_{1}+k, n}+\beta_{02} \theta_{2}^{N_{1}+k-1, n}+\beta_{03} W_{2}^{N_{1}+k, n}, \quad n=0,1, \ldots, N_{t}-1, k=1,2, \ldots, N_{2}-1 \text {, }
$$

where,

$$
\theta_{1}^{N_{1}+k, n}=\theta_{1}\left(y_{N_{1}+k}, t_{n}\right) \text {, and, } \beta_{00}=\beta_{02}=\frac{\Delta t}{Q_{2} \Delta y_{2}^{2}}, \beta_{01}=1-\frac{2 \Delta t}{Q_{2} \Delta y_{2}^{2}}, \beta_{03}=\frac{\Delta t}{Q_{2}} .
$$

Using the boundary condition $(84)_{2}$, we obtain

$$
\theta_{2}^{N_{1}+N_{2}, n}=1, \quad n=1,2, \ldots, N_{t} .
$$

Now, the above relations, determine the values $\theta_{1}\left(y_{j}, t_{n}\right), \theta_{2}\left(y_{k}, t_{n}\right)$ of the temperature fields.

Indeed, using Eqs. (93) and (95), the values $\theta_{2}^{N_{1}+k, n}, k=1,2, \ldots, N_{2}, n=0,1, \ldots, N_{t}$ are determined.

The values $\theta_{1}^{j, n}, j=0,1, \ldots, N_{1}, n=0,1, \ldots, N_{t}$ of the temperature field in zone $y \in[0,1]$ are determined using Eq. (87) together with Eq. (92).

\section{Numerical results and discussion}

Flows inside channels, such as magneto-hydrodynamic, electro-hydrodynamic, and electrokinetic flows are important in many microscale applications. The generation of such flows is often done with the help of external fields (mechanical, thermal, magnetic, electric) applied to the fluids. In this section, the generalized Couette flow studied in previous paragraphs is numerically and graphically simulated in the case when the motion of the upper plate is given by the function $f(t)=1-\exp (-t), t \geq 0$.

For the non-dimensional parameters that characterize the studied fluids and fields, the following numerical values were used: $d=1.5, \omega=\pi / 4, F_{1}=0.5, F_{2}=0.6, E_{1}=0.55, E_{2}=0.75, R e_{1}=0.8, R e_{2}=2$, $R m_{1}=1.2, R m_{2}=0.8, P r_{1}=1.5, P r_{2}=3.5, E c_{1}=2.5, E c_{2}=1.5, G_{0}=1.5, H b_{1}=0.8, H b_{2}=0.4, \gamma_{0}=\pi / 6$.

Figure 2 shows the profiles of fluid velocities in both flow regions for different values of the time t. As expected, the fluid flows more slowly in the region bounded by the lower wall. This behavior is due to the fact that the lower wall of the canal is fixed, so the viscosity force will slow down the fluid motion. In the region of the canal bounded by the upper wall, the fluid moves at a higher velocity due to the movement of the wall with the velocity given by the function $f(t)$. It is observed in Fig. 2 that for large values of the time $t$, fluid velocity on the upper wall becomes almost constant, because $\lim _{t \rightarrow \infty} f(t)=\lim _{t \rightarrow \infty}(1-\exp (-t))=1$.

This property is most clearly evident from Fig. 3 which shows the profiles of velocities as a function of time $\mathrm{t}$ for three positions of the channel, namely, $y \in\{0.5,1.0,1.25\}$. The curves in Fig. 2 , show that for $t \geq 15$ fluid velocities tend to become constant. Also, Figs. 2 and 3 highlight that fluid velocity satisfy the interface condition $u_{1}(1, t)=u_{2}(1, t)$.

Figures 4 and 5 show profiles of the ratio of induced magnetic field, and the external applied magnetic field, versus spatial coordinate $y$, respectively versus time $t$. Note that in the $y=1$ position of the channel, where the velocities are equal, the variation of the induced magnetic field is slower. It can be seen that in this position the induced magnetic field has a slow increase and after the value $t=5$, the values of the induced magnetic field are almost constant. In the first flow zone, in which the velocity gradient has large variations, the induced magnetic field also has significant variations. These variations are attenuated in the second flow area where the speed gradient variations are smaller. Also, we note that for $t \geq 15$, the values of induced magnetic field become almost constant for any position of the channel. This behavior is in accordance with the fluid motion whose velocity tends to constant values for large values of the time $t$.

Figures 6 and 7 are plotted to show the variation in space and time of the dissipative functions $W_{1}(y, t), W_{2}(y, t)$. It is known that viscous dissipation acts as a source of energy in the fluid flow and it affects the temperature distribution. The shear stress within the fluid layer induced by the motion of the upper plate significantly influences the energy dissipation. It is observed in Figs. 6 and 7 that for a short time interval, the dissipative function in the region bounded by the lower wall is higher than the dissipative function in the other region. After this instant, the dissipative function in the region bounded by the upper plate becomes higher. This fact is generating by the evolution in time of the velocity in this region.

\section{Conclusions}

Unsteady, magneto-hydrodynamic generalized Couette flows of two immiscible fluids in a rectangular channel have been studied. 

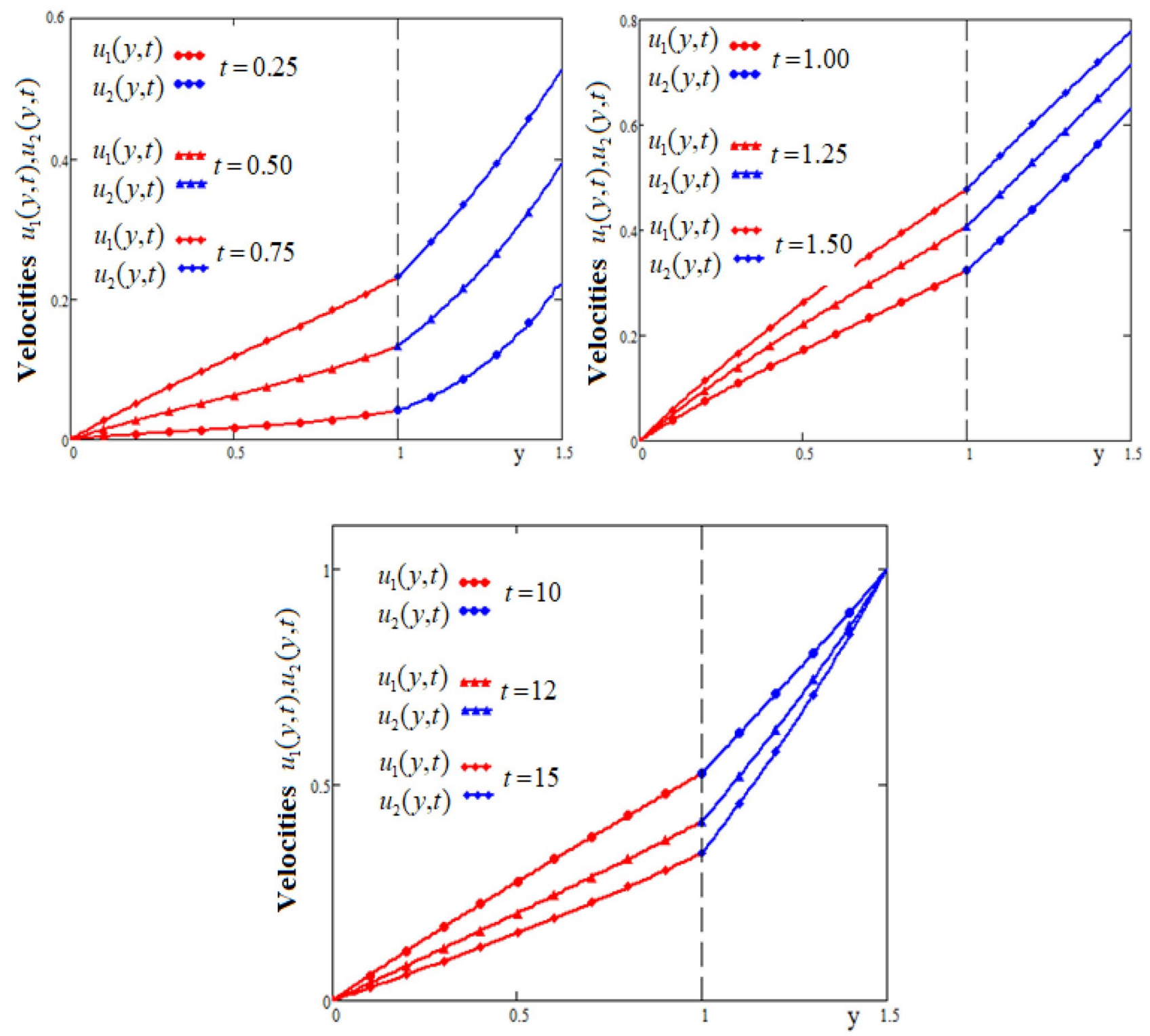

Figure 2. The profiles of velocities $u_{1}(y, t), u_{2}(y, t)$ versus $y$ for different values of the time $t$.

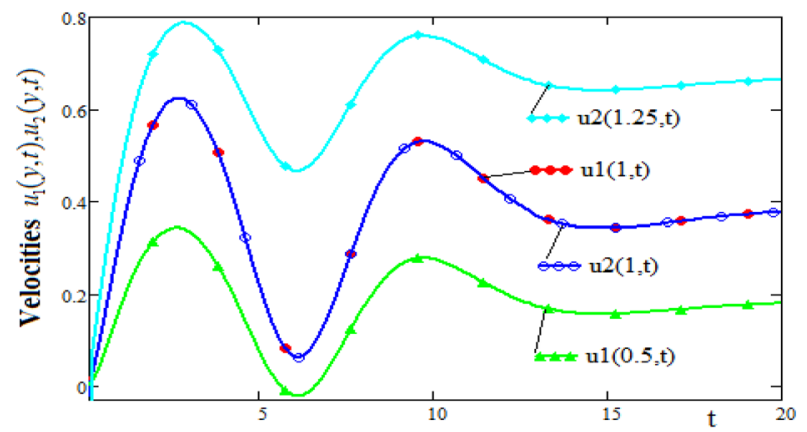

Figure 3. Time-evolution of velocities $u_{1}(y, t), u_{2}(y, t)$ in three different positions of channel. 

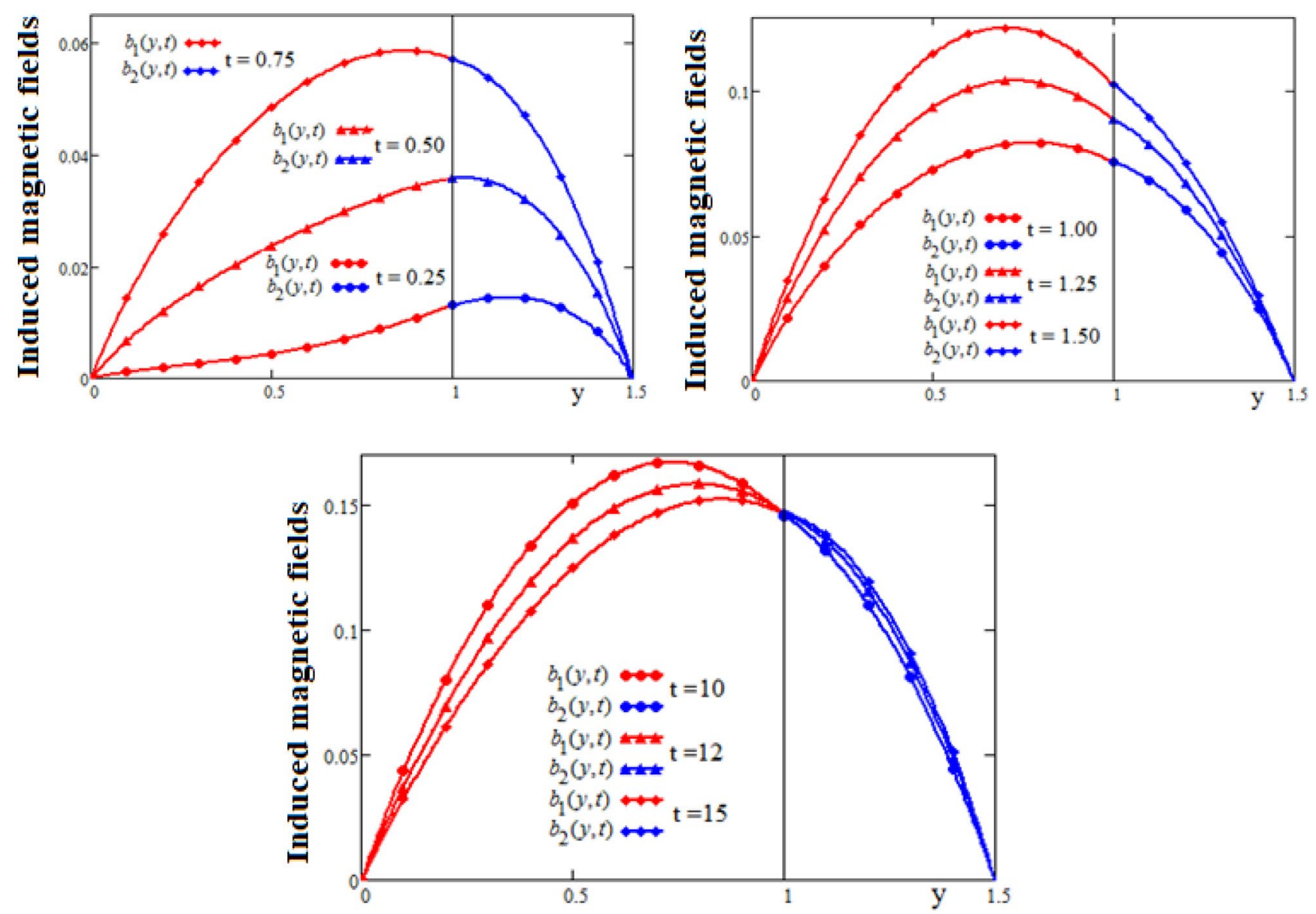

Figure 4. The profiles of induced magnetic fields $b_{1}(y, t), b_{2}(y, t)$ versus $y$ for different values of the time $t$.

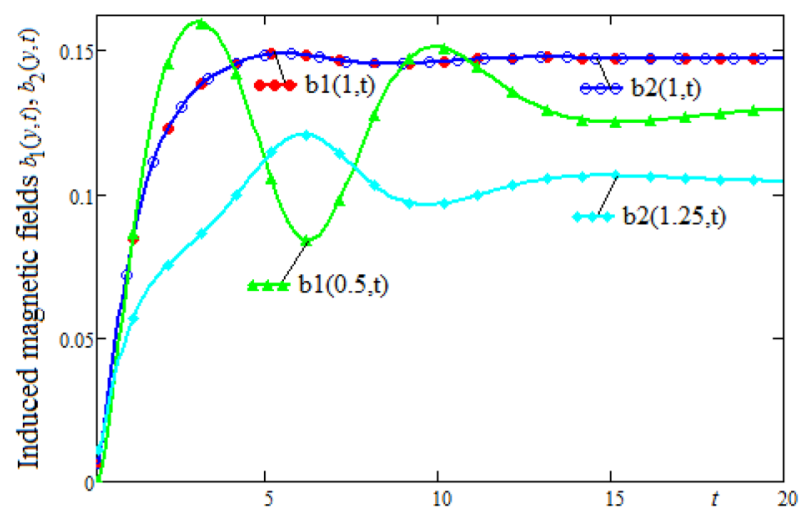

Figure 5. Time-evolution of induced magnetic fields $b_{1}(y, t), b_{2}(y, t)$ in three different positions of channel.

The flow is influenced by an inclined magnetic field and an axial time-oscillating electric field. Both fluids are considered electrically conducting and the solid boundaries are electrically insulated.

The problem is formulated into the dimensionless form and at the interface the velocity, shear stress, induced magnetic and temperature fields are considered continuous functions. Both walls are isothermal. The bottom wall of channel is fixed, while the upper wall is moving with a given time-dependent velocity. The nonslip conditions on the solid boundaries are also considered.

Approximate analytical solutions for the velocity, induced magnetic, and temperature fields have been determined using the Laplace transform method along with the numerical Stehfest's algorithm for the inversion of the Laplace transforms.

A numerical scheme based on the finite differences has been developed for the non-linear energy equation. 

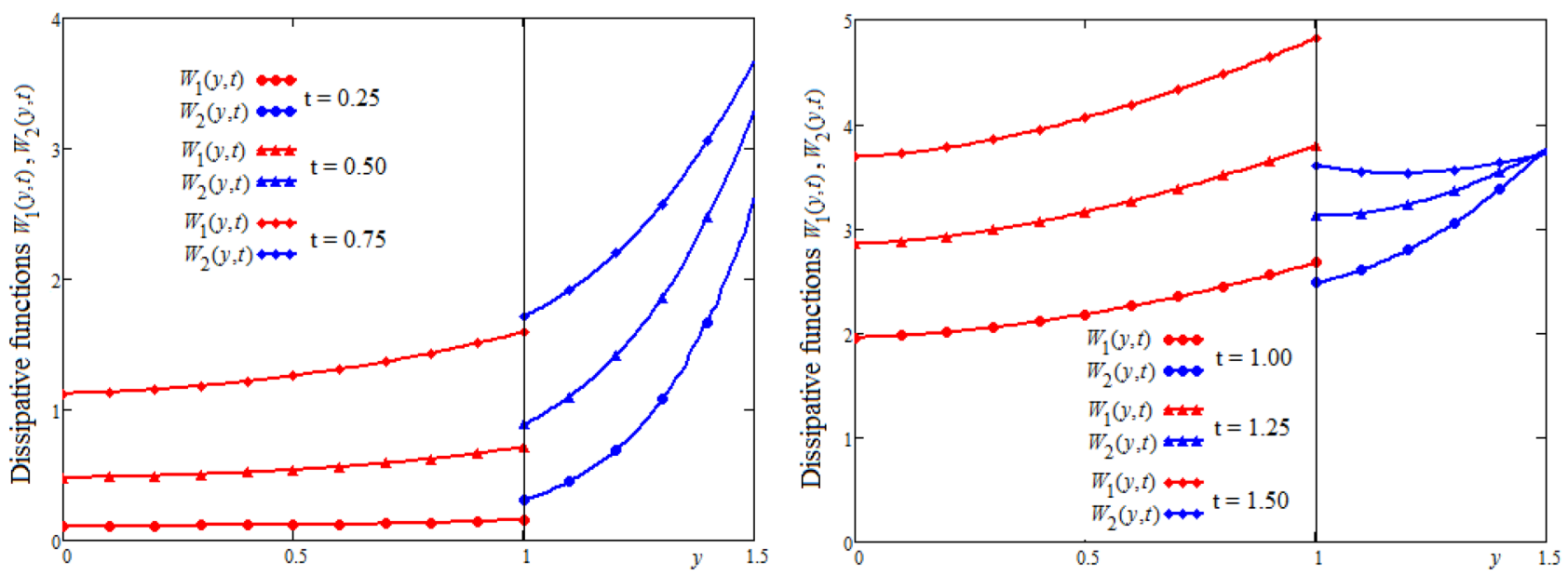

Figure 6. The profiles of dissipative functions $W_{1}(y, t), W_{2}(y, t)$ versus $y$ for different values of the time $t$.

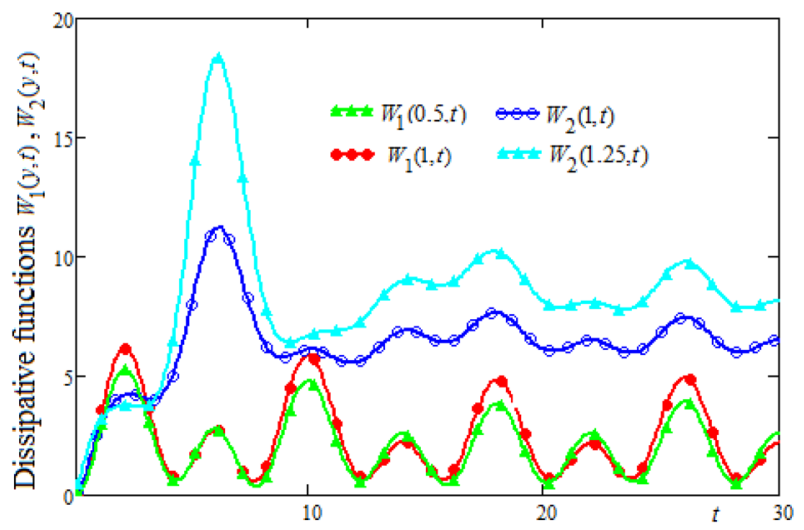

Figure 7. Time-evolution of the dissipative functions $W_{1}(y, t), W_{2}(y, t)$ in three positions of channel.

A particular case characterized by a time-exponential velocity of the upper wall, has been numerically and graphically studied to show the evolution of the fluid velocity, induced magnetic field, and viscous dissipation in both flow regions.

Received: 9 June 2021; Accepted: 22 November 2021

Published online: 07 January 2022

\section{References}

1. Adamson, A. Physical chemistry of surfaces (Mir, 1979) (in Russian)

2. Shum, H. C., Sauret, A., Fernandez-Nieves, A., Stone, H. A. \& Weitz, D. A. Corrugated interfaces in multiphase core-annular flow. Phys. Fluids 22, 082002 (2010).

3. Blum, E. L., Zaks, M. V., Ivanov, U. I. \& Mikhailov, Y. A. Heat exchange and mass exchange in magnetic field. Zinatne Riga 223, 1 (1967).

4. Cramer, K. R. \& Pai, S. I. Magnetofluid dynamics for engineers and applied physicists (McGraw-Hill, 1973).

5. Soundalgekar, V. M., Vighnesam, N. V. \& Takhar, H. S. Hall and ion slip effects in MHD Couette flow with heat transfer. IEEE Trans. Plasma Sci. 7(3), 178-182 (1979).

6. Attia, H. A. Hall current effects on the velocity and temperature fields of an unsteady Hartmann flow. Can. J. Phys. 76(9), 739-746 (1998).

7. Thome R. J. Effect of transverse magnetic field on vertical two- phase flow through a rectangular channel. Argonne National Laboratory Report No. ANL (1964) 6854. https://doi.org/10.2172/4046687.

8. Postlethwaite, A. W. \& Sluyter, M. M. MHD heat transfer problems-an overview. ASME Mech. Eng. 100, 32-39 (1978).

9. Lohrasbi, J. \& Sahai, V. 'Magnetohydrodynamic heat transfer in two-phases flow between parallel plates. Appl. Sci. Res. 45, 53-66 (1988).

10. Malashetty M. S., \& Leela V. Magnetohydrodynamic heat transfer in two fluid flow. In Proceedings of the National Heat Transfer, Conf. AIChE \& ASME, HTD (1991), p 159.

11. Malashetty, M. S. \& Leela, V. Magnetohydrodynamic heat transfer in two phase flow. Int. J. Eng. Sci. 30, 371-377 (1992).

12. Malashetty, M. S. \& Umavathi, J. C. Two-phase magnetohydrodynamic flow and heat transfer in an inclined channel. Int. J. Multiphase Flow 23, 545-560 (1997).

13. Sai, K. S., Swamy, N. S., Nataraja, H. R., Tiwari, S. B. \& Rao, B. N. Unsteady flow of two immiscible fluids under an oscillatory time-dependent pressure gradient in a channel with one porous floor. Trends Appl. Sci. Res. 1(2), 194-203 (2006). 
14. Pasquier, S., Quintard, M. \& Davit, Y. Modeling two-phase flow of immiscible fluids in porous media: Buckley-Leverett theory with explicit coupling terms. Phys. Rev. Fluids. 2(10), 1-19 (2017).

15. Nikodijevic, D., Stamenkovic, Z., Milemkovic, D. \& Blagojevic, B. Flow and heat transfer of two immiscible fluids in the presence of uniform inclined magnetic field. Math. Probl. Engin. 1, 1. https://doi.org/10.1155/2011/132302 (2011).

16. Luo, L., Shah, N. A., Alarifi, I. M. \& Vieru, D. Two-layer flows of generalized immiscible second grade fluids in a rectangular channel. Math. Methods Appl. Sci. 43(3), 1337-1348 (2019).

17. Rauf, A., Mahsud, Y. \& Siddique, I. Multi-layer flows of immiscible fractional Maxwell fluids in a cylindrical domain. Chin. J. Phys. 67, 265-282 (2020).

18. Rauf, A., Rubbab, Q., Shah, N. A. \& Malik, K. R. Simultaneous flow of n-immiscible fractional maxwell fluids with generalized thermal flux and robin boundary conditions. Adv. Math. Phys. 20, 1 (2021).

19. Gaikwad, H. S., Baghel, P., Sarma, K. \& Mondal, P. K. Transport of neutral solutes in a viscoelastic solvent through a porous microchannel. Phys. Fluids 31(2), 2006 (2019).

20. Gaikwad, H. S. \& Mondal, P. K. Rheology modulated high electrochemomechanical energy conversion in soft narrow-fluidic channel. J. Non-Newton. Fluid Mech. 285, 4381 (2020).

21. Sarma, R. \& Mondal, P. K. Thermosolutal Marangoni instability in a viscoelastic liquid film: Effect of heating from the free surface. J. Fluid Mech. 909, 1 (2021).

22. Sarma, R., Jain, M. \& Mondal, P. K. Towards the minimization of thermodynamic irreversibility in an electrically actuated microflow of a viscoelastic fluid under electrical double layer phenomenon. Phys. Fluids 29, 103102 (2017).

23. Sarma, R., Nath, A. J., Konwar, T., Mondal, P. K. \& Wongwises, S. Thermo-hydrodynamics of a viscoelastic fluid under asymmetrical heating. Int. J. Heat Mass Transf. 125, 515-524 (2018).

24. Balasubramanian, S., Kaushik, P. \& Mondal, P. K. Dynamics of viscoelastic fluid in a rotating soft Microchannel. Phys. Fluids 32, 112003 (2020).

25. Stehfest, H. Algorithm 368: Numerical inversion of Laplace transforms. Commun. ACM 13(1), 47-49 (1970).

26. Davies, B. \& Martin, B. Numerical inversion of the Laplace transform: A survey and comparison of methods. J. Comput. Phys. 33(1), 1-32 (1979).

\section{Acknowledgements}

This work was conducted under the Technology Innovation Program (or Industrial Strategic Technology Development Program-material part package type) as "Development of fire suppression-type high safety module and demonstration of safety for future eco-friendly medium and large secondary battery (No. 20015986)", funded by the Ministry of Trade, Industry \& Energy (MOTIE), Republic of Korea.

\section{Author contributions}

N.A.S., H.A. and D.V. prepared the literature survey, references section, constructed analytic solutions and sketched the graphs. D.V. and S.J.Y. implemented a software package and calculated the tabulated values. D.V., Supervision and S.J.Y, Funding, All the authors have read and approved the final draft of the manuscript.

\section{Competing interests}

The authors declare no competing interests.

\section{Additional information}

Correspondence and requests for materials should be addressed to S.-J.Y.

Reprints and permissions information is available at www.nature.com/reprints.

Publisher's note Springer Nature remains neutral with regard to jurisdictional claims in published maps and institutional affiliations.

Open Access This article is licensed under a Creative Commons Attribution 4.0 International License, which permits use, sharing, adaptation, distribution and reproduction in any medium or format, as long as you give appropriate credit to the original author(s) and the source, provide a link to the Creative Commons licence, and indicate if changes were made. The images or other third party material in this article are included in the article's Creative Commons licence, unless indicated otherwise in a credit line to the material. If material is not included in the article's Creative Commons licence and your intended use is not permitted by statutory regulation or exceeds the permitted use, you will need to obtain permission directly from the copyright holder. To view a copy of this licence, visit http://creativecommons.org/licenses/by/4.0/.

(C) The Author(s) 2022 